\title{
THE ROUTINE DETECTION AND ESTIMATION OF BORIC ACID IN BUTTER.
}

\author{
By HERBERT HAWLEY, M.Sc., F.I.C.
}

(Read at the Meeting, March 3, 1915.)

THE usual methods for the estimation of boric acid in butter involve remoral of the fat, and titration of the acid in the presence of glycerol or mannitol. Though perfectly satisfactory when an accurate estimation of the preservative is required, or for a single sample, the process is somewhat laborious when it has to be applied to a large series of samples. The majority of such samples are either free from boric acid or contain an allowable proportion, the detection and an approximate estimation of which is generally all that is required.

The following process gives reliable approximate estimations of the boric acid; it has the advantage that (1) the portion used for the examination of the fat serves also for both detection and estimation, and (2) a number of samples can be examined together in a very short time.

The following reagents are required :

Turmeric Reagent.-A mixture of 5 grms. powdered turmeric root and 5 grms. tartaric acid is digested with three successive portions of 150 c.c. warm alcohol (industrial methylated spirit is quite satisfactory). Each digestion should continue for not less than an hour, and each successive portion should be filtered, and the volume finally made up to 500 c.c. with alcohol. (This reagent should be kept in the dark.)

Dilute Hydrochloric Acid.-Twenty c.c. concentrated hydrochloric acid are diluted to a litre with water.

One Per Cent. Standard Boric Acid Solution.-One grm. boric acid is dissolved in 100 c.c. of the dilute hydrochloric acid.

Milk.-For convenience, a sample may be preserved with formaldehyde, which is without effect on the test.

Method of Working.-Twenty grms. of each sample of butter are placed in small beakers of uniform size of about 40 to 50 c.c. capacity. The butter may be weighed 
in a rough balance, or, more rapidly, the apparatus described at the end of this paper may be used to deliver the sample. The samples are melted in the usual way on a copper tray over either a water-bath or steam oven, and kept warm until the curd and aqueous liquid have separated, leaving the fat clear. The fats are now poured off, as far as possible, on to filter-papers placed in small beakers (care must be taken that none of the aqueous portion of the butters is poured on to the filters), and the constants of the filtered fats examined by the usual methods. To the aqueous residues in the beakers 18 c.c. of the dilute hydrochloric acid are added from a small measuring cylinder. The contents of the beakers are stirred and kept warm for a few minutes. Allowing 2 c.c. for the water in the butter, we now have approximately 20 c.c. of dilute acid containing any boric acid originally present in the 20 grms. of butter, together with curd and a little fat floating on the top of the liquid.

Ten c.c. of fat-free liquid are removed from each beaker by means of a pipette dipped through to the bottom of the liquid. While the liquid is held in the pipette, the residue (fat and aqueous liquid) in the beaker is rejected. The liquid in the pipette is then returned to the beaker. This gives 10 c.c. of an opalescent liquid, which should be almost free from fat, though containing a little curd. The beakers are allowed to cool, and to each is added 5 c.c. of the turmeric reagent. Those samples which contain boric acid slowly develop a reddish-brown colour, the intensity varying with the amount of boric acid present. To estimate approximately the boric acid, standards prepared as follows are put on at the same time: Two burettes are required, one containing 1 per cent. boric acid solution, and the other dilute hydrochloric acid. Standards representing $0,0.1$ per cent., 0.3 per cent., and 0.5 per cent. $\mathrm{H}_{3} \mathrm{BO}_{3}$ in the original butter will be found convenient, and are prepared thus :

0.0 per cent. 9.5 c.c. dilute $\mathrm{HCl}$, and 0.5 c.c. milk.

$0 \cdot 1 " 1$ c.c. standard boric solution, $8 \cdot 5$ c.c. dilute $\mathrm{HCl}, 0.5$ c.c. milk.

$0.3 ", 3 \quad ", \quad 6.5$

$0.5 \quad " \quad 5 \quad \%, \quad ", 4.5$

$4.5 \quad " \quad "$

To each are added 5 c.c. of the turmeric reagent. The milk gives a slight opalescence, making the comparison of the standards and samples more satisfactory. The comparison should be made not less than one and a half hours, not more than three hours after the addition of the turmeric. The colour develops gradually, and after a few hours commences to fade. The reagent gives an unmistakable colour when 0.05 per cent. boric acid is present in the butter, and the percentage up to 0.5 per cent. can be estimated with certainty to the nearest 0.1 per cent. Should any samples be found to contain more than 0.5 per cent. boric acid, it is best to determine the quantity by extraction and titration in the usual manner, as with such quantities the colour becomes more intense, but the gradation is not so good.

This process has been used for the routine examination of butter in this laboratory for the past twelve months. The results obtained have been frequently checked by estimation in the ordinary way. The agreements have always been good.

The apparatus I have devised will be found useful for measuring out the 20 grms. 
of butter. It consists of a stout piece of glass tubing of about $\frac{7}{8}$ inch bore and 6 inch length. A plunger is easily made by boring a hole nearly through a solid rubber bung which will just slide into the tube. A piece of narrow glass tubing with a bulb on the end, and sealed at the other end, is then inserted in the hole, and string tied tightly round the narrow end of the bung. To take a sample, the open end of the tube is pressed into the butter, at the same time lifting the plunger. The latter is then pressed down to the mark (which is adjusted so that the tube then contains 20 grms. of butter), and the butter pressed out into the beaker. It will be found advisable to cover the inner tube with indiarubber tubing, otherwise when the sample is delivered the inner tube may strike the outer and cause breakage.

City axalyst's Laboratory, Birmingham. 